\title{
4
}

\section{Laser Scanner: eSafety \& ITS Applications}

\author{
Nieves Gallego Ripoll \\ Universidad Politécnica de Valencia \\ Spain
}

\section{Introduction}

This chapter describes a proposal for a new type of Laser Scanner for use in traffic management. The chapter begins by outlining current traffic management systems, ongoing research areas and the perceived failures of present and some proposed implementations. Though the initial background presentation is long winded this is deemed necessary so the reader can gain a fuller insight into the new Laser Scanner technology being proposed.

The United Nations on its report "State of world population 2007" (UNFPA, 2007) declared that:"In 2008, for the first time, more than half of the world's population will be living in urban areas. By 2030, towns and cities will be home to almost 5 billion people". This growth both in cities and inhabitants is directly proportional to mobility demands and requirements. In this situation, strategic traffic management is a key factor, even though it also involves different sectors such as economic (oil dependency), environmental (pollution, noise...), safety (both drivers and pedestrians) and health (breathing problems, circulation...).

Traffic authorities are encouraged to coordinate traffic management and monitoring focusing on Road Safety problems. The Intelligent Transport Systems (ITS) is a solution in traffic management and user mobility thanks to the development and use of different applications and technologies (Ertico, 2008; ITS, 2008). Intelligent Transport Systems and Services include a wide variety of sectors and areas that previously did not have a direct connection between them.

ITS systems increase road safety by incorporating breakthrough technologies in different levels of management and control. These subsystems (traffic flow information systems, travelers' information systems, highways monitoring and management systems, incident management, pedestrian detection...) rely on the traffic parameters provided by sensors measuring in real-time the situation on the road. The quality of the metrics received is a key for a correct network management and monitoring (García, 2000; Martin et al., 2003; Van Arem et al., 1993). The Utah Traffic Laboratory (UTL) on the report "Detector Technology Evaluation" carried out in November 2003 specifies that: "The data collected must be plentiful, diverse, and accurate. These complex data requirements present a challenge to traffic detection systems"(Martin et al., 2003). This statement demonstrates the importance of the traffic data and therefore of the sensor systems.

Traditionally, sensors have been used as mere measurement points, isolated from each other, that send the information to a central node which gathers the intelligence and information generated by the system (Sepulcres \& Gozálvez, 2006). This node supports the applications, 
the processing and analysis of data received and also, in certain cases, executes the required remedial actions or control strategy.

Since there are a number of sensing technologies relevant to ITS, several studies and reports comparing them have been published (Fang et al., 2007; Klein et al., 2006; Mimbela \& Klein, 2007; Skszek, 2001; Turner \& Austin, 2000). Inductive loops and magnetic sensors require road closure for their installation and maintenance. The video image processor is vulnerable to weather and light conditions. Ultrasonic and infrared sensors lack stability in noisy environments. Microwave radars are not suitable for vehicle classification (Klein et al., 2006). Laser scanner sensors installation and maintenance do not require road closure, and are more robust and less vulnerable to weather conditions and noisy environments, and are thus the best option for traffic parameter detection.

To solve this situation, a solution is presented to optimize infrastructure sensors already installed in cities, focusing on the case of laser scanners, tackling the problem in two ways: on one hand by developing sensors able to provide more accurate and valuable traffic parameters and on the other by endowing the sensors with "intelligence". These innovations introduce an approach geared towards a new generation of sensors able to cooperate and communicate with the traffic control center (TCC).

The detection system used is a laser scanner, which provides information in real-time about: detection (presence), flow rate, counting and vehicle classification. The classification has been made following statistical methods in eight groups $(8+1)$ : powered two-wheels (motorbikes, ... ), car, car with trailer, van, truck, truck with trailer, artic, bus + unclassified. The intention is to improve traffic management quality by providing supported information about the traffic parameters on the road network.

\subsection{State of the art}

Road safety became a global concern with the release of the, "World Report on Road Traffic Injury Prevention" (Peden et al., 2004). This report declares that, "Of all the systems with which people have to deal every day, road traffic systems are the most complex and the most dangerous. Worldwide, an estimated 1.2 million people are killed in road crashes each year and as many as 50 million are injured. Projections indicate that these figures will increase by about $65 \%$ over the next 20 years unless there is new commitment to prevention". Some important mistakes were detected, "Insufficient attention to the design of traffic systems" (Peden et al., 2004). This statement confirms the reason to develop and design traffic monitoring systems to help reduce the actual traffic accident rate.

In April 2004, the General Assembly of the United Nations (UN) approved a resolution urging the improvement of road safety in the world (UN, 2004). This assembly invited the World Health Organization (WHO) to coordinate the safety activities within UN organizations. This was the beginning of the, "Road Safety Collaboration Group" with representatives of more than 42 organizations. Key provisions of the UN resolutions related with "Road Safety" are summarized in:

- May 2003 - UN General Assembly: Global Road Safety crisis. Called on governments and civil society to raise awareness and enforce existing road safety legislation (UN, 2003b).

- November 2003 - UN General Assembly: Global Road Safety crisis. Formalized, "Improving road safety" as an agenda item for the 60th General Assembly Session (UN, 2003a). 
- May 2004 - UN General Assembly: Improving Road Safety. Acknowledged the release of the World Report on Road Traffic Injury Prevention (UN, 2004).

- May 2004 - World Health Assembly: Road Safety and Health. Requested to encourage research to support evidence-based approaches for prevention of road traffic injuries and mitigation of their consequences (WHA, 2004).

- October 2005 - UN General Assembly: Improving Global Road Safety. Encouraged international community to lead financial, technical and political support to improve road safety (UN, 2005).

- March 2008 - UN General Assembly: Improving Global Road Safety. Encouraged private and public sector to implement policies to reduce crash risk for occupants and other road users (UN, 2008).

In this situation, strategic traffic management is a key factor in Intelligent Transport Systems applications. In urban areas, the traffic control center gathers parameters and information about real time traffic which are used to implement traffic policies and legislation.

\subsection{Review of related work: Laser Scanner in ITS systems}

In the literature, three main research lines are the most important: sensor development, applications and improvement of time-of-flight (ToF) techniques. Today, most of the effort is focused on development of the applications. The group of authors working on sensor development focuses on laser optical systems and laser telemetry. In the University of California-Davis, Cheng et al.(Cheng et al., 2005; 2001) developed a laser-based detector using a laser line projected on the ground and the reflected beam is collected and focused onto a photodiode array. Even though this sensor can provide information at speed, it uses two lasers. The weakness of the system is sensor costs are increased by using two transmitters and receivers. A slight error in the laser beam alignment during the installation of the sensors can result in significant errors and further, only one lane can be monitored at a time. The range sensor presented by the University of Central Florida cannot be applied in real-time (Hussain \& Moussa, 2005).

Other research avenues consider it more important to use well designed and highly reliable commercial products than to work on the development of new sensors and work on improving acquisition and signal treatment without going into sensor technology. This group focuses on the application and represents the majority of the current research (Abdelbaki et al., 2001; Fuerstenberg \& Dietmayer, 2004; Gallego et al., 2007; Harlow, 2001; Hussain \& Moussa, 2005). Fuerstenberg and Dietmayer (Fuerstenberg \& Dietmayer, 2004) investigate sensors installed in vehicles in order to detect moving pedestrians. Hussain and Moussa (Hussain \& Moussa, 2005) develop a vehicle classification system called AVCSLII. The classification algorithm was produced by training neural networks and experimental results present five classes based on a database of 4995 vehicles. Harlow and Peng (Harlow, 2001) propose a solution to vehicle detection by processing range imagery. The solution produces different classification methods generated by two laser scan lines separated by $10^{\circ}$.

The authors do not take advantage of the two sweeping laser scan lines to exploit all the potential information, nor do they use the laser scans to measure vehicle speed. The proposal lacks in-depth analysis of speed, requires a larger database and further study under different weather conditions. Lastly, scientists have made a breakthrough in circuit design. It is worth mentioning the research of J. Kostamovaara of the University of Oulu in Helsinki (Palojarvi 
et al., 2005; Pehkonen et al., 2006), who investigated and optimized the receiver channel for a pulsed, ToF, laser range finder using BiCMOS technology.

\section{System description}

Present Laser Scanners are not designed specifically for traffic control applications, though the few which are do not provide the software needed for signal acquisition and treatment thus none of the actual systems can provide information in real time.

The proposed Laser Scanner System provides a set of sensor (laser scanner), communications and software modules able to detect and classify vehicles in real-time, acquiring and storing their silhouette and 3D image in addition to required traffic parameters.

\subsection{Technical requirements}

Laser Scanners measure the ToF of the coherent light coming from an emitter laser, and reflected by the vehicle for its detection. By means of the laser technology, parallel and coherent beams can be generated. A pulsed laser beam is emitted by the laser scanner and reflected by the vehicle. The reflection is registered by the scanner receiver. The time elapsed between the emission and reception of the pulse is directly proportional to the distance between the scanner and the object. For correct vehicle detections the minimum number of readings to identify a vehicle is 20 measurements. Laser scanners measure the time-of-flight of the laser pulse reflected by the vehicle. The laser works in the infrared range, approximate wavelength $0,9 \mathrm{um}$, which is outside the visible range thus avoiding possible distraction to road users. Furthermore the chosen laser frequency also lies outside the bandwidth of sunlight thereby minimizing this source of interference.

The laser scanner is located at a suitable height above the road to cover all lanes. Therefore the system must be located vertically above of the road with a minimum height of 5 meters, Fig. 1 (a). In order to provide the required cross-sectional readings on each vehicle, it is advised to work with angular steps of $1^{\circ}$, Fig. 1(b). This angle can be increased as long as direct vision to the lanes is guaranteed.

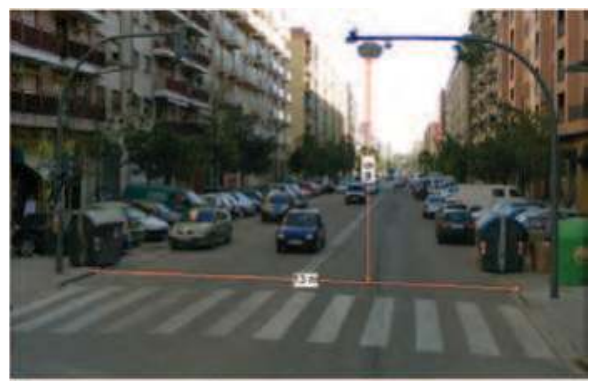

(a) Laser Scanner Positioning

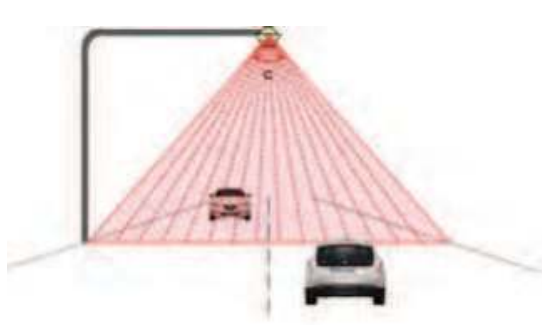

(b) Operation Principle

Fig. 1. Operation Principle

The communications between sensor and control unit included in the laser scanner system will be done via RS232 using a proprietary protocol. This communication can be implemented using the Ethernet protocol. By means of these specifications, the laser system should be able to detect and classify vehicles; as well as, acquire and store their silhouette. 
After the preliminary studies carried out laser scanner system requirements for its use are shown in Table 1.

\begin{tabular}{|c|c|}
\hline \multicolumn{2}{|c|}{ System Requirements } \\
\hline \multicolumn{2}{|l|}{ General } \\
\hline \multicolumn{2}{|c|}{ located vertically above of the road $(\mathrm{m}) 5$} \\
\hline Maximum Consumption (W) & 40 \\
\hline Laser Class & I (safe) \\
\hline Emitter type & IR Laser diode \\
\hline \multicolumn{2}{|l|}{ Communications } \\
\hline Interface & RS232 optional RS485/422 \\
\hline Baudrate (KBd) & $9,6-500$ \\
\hline High speed option (MBd) & 1,5 \\
\hline \multicolumn{2}{|l|}{ Scanner } \\
\hline Scanning frequency (HZ) & $15-100$ \\
\hline Response time (ms) & $53-10$ \\
\hline Scanning angle $\left(^{\circ}\right)$ & $100-180$ \\
\hline Angular resolution $\left(^{\circ}\right)$ & $0,25-1$ \\
\hline Resolution $(\mathrm{mm})$ & 10 \\
\hline \multicolumn{2}{|l|}{ Functional } \\
\hline \multicolumn{2}{|c|}{$\begin{array}{l}\text { The laser scanner system should be able to detect and classify vehicles } \\
\text { The laser scanner system should be able to allow the acquisition and storage of } \\
\text { silhouette of vehicles } \\
\text { The laser scanner system should be located in the vertical of the road to a minimum } \\
\text { altitude of } 5 \text { meters } \\
\text { The laser scanner system must have direct vision to the lanes } \\
\text { The laser scanner system should be able to communicate to the local traffic control } \\
\text { center using Ethernet Communication }\end{array}$} \\
\hline
\end{tabular}

Table 1. Technical Requirements

\subsection{Laser Scanner measurement and data principle}

Laser scanner is used in measuring mode (of the distance values). This means that the laser scanner outputs each distance value in two data bytes. In the standard measuring configuration, data bits 0 to 12 are used to represent the distance, while data bits 13 to 15 are not used. These 13 data bits enable $213-1=8191$ coding options to be represented. As we have selected a measured value resolution of $1 \mathrm{~mm}$, this will result in a maximum measuring distance of $8191 \mathrm{~mm}$.

The communications with the laser scanner are done through commands. Commands are a set of predefined instructions which carry out different operations like the configuration of the system. The following remarks are important to know how the laser scanner communicates, thus it is also important to understand the hardware developed:

- Data format is set as follows: 1 start bit, 8 data bits and 1 stop bit (8N1). 
- Laser scanner outputs data in ascending angular steps. The angular values themselves are not transmitted; instead, the data field only comprises distance values.

- Laser scanner is configured by means of commands called telegrams. A send telegram always contains only one control command, and a response telegram contains only one response from the laser scanner.

The next instructions set must be followed in order to configure the laser scanner: baudrate configuration $(9600,38400$ or $500 \mathrm{~K})$, range configuration $\left(100^{\circ}, 180^{\circ}\right)$, resolution $\left(1^{\circ}, 0.5^{\circ}\right.$, $\left.0.25^{\circ}\right)$ and measurement units $(\mathrm{mm}, \mathrm{cm})$.

\subsection{Architecture hardware}

The detector is divided into three parts: the sensor, the control unit, and the software classification program (Gallego et al., 2009). The sensor is installed in the pole of the traffic light. The control unit comprises the hardware and communications module. And lastly, the classification program running on a computer.

The communications between the sensor and the control unit is via RS422. At high speed baudrate communication it is not possible to use serial communication between the control unit and the computer, therefore we have chosen standard USB. This way connection to the computer would be effected using the USB port.

The control unit must: establish communication with the laser scanner, modify the configuration settings and carry out the signal pre-treatment using DSP microcontrollers (Digital Signal Processor). The signal processing is done in two stages which are in different physical locations. The vehicle detection is done on the control unit and the vehicle classification is done on the computer. The final control unit designed is shown in Fig. 2.

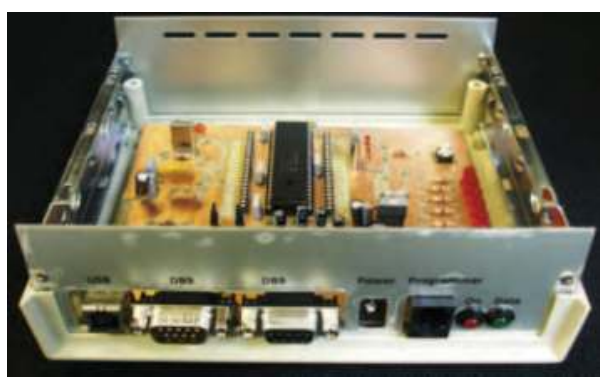

(a) HW front

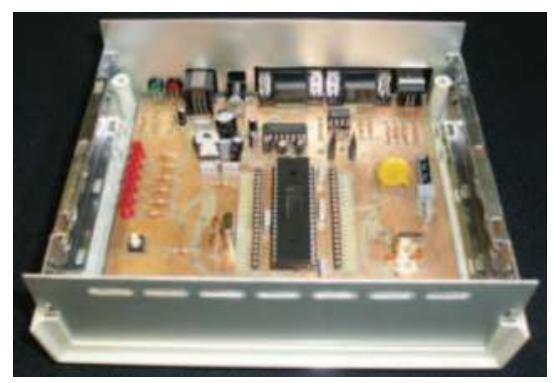

(b) HW back

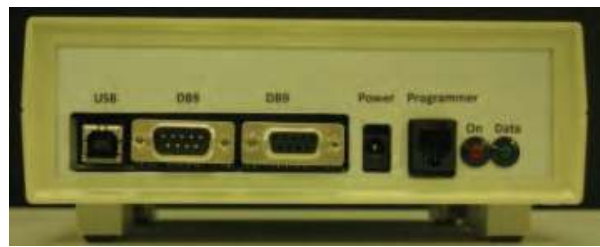

(c) HW connectors

Fig. 2. Hardware Design 
The first task was to establish communication with the laser scanner sensor. The sensor sends data using the communication standard RS422; it provides a service for data transmission, using balanced or differential signaling. Therefore the first requirement was to develop hardware able to read this information using the computer serial port. For this purpose the hardware unit designed included differential drivers and converters. The differential driver and receiver pair was designed for balanced transmission-line and it is used to convert from RS422-TTL. Later on, another converter from TTL to RS232 level is needed to communicate using the serial port of the computer. The communication was established successfully with the laser scanner sensor. The bidirectional communication required for setting the configuration was facilitated by means of the sensor commands.

DSP microcontroller is the only solution, because the sensor sends 361 measurements per scan each 13 ms. The DSP firmware was developed for:

- signal pre-treatment,

- laser scanner configuration,

- and data configuration.

\section{Data normalization}

The signal processing is done in two stages which are in different physical locations. The vehicle detection is done on the DSP and the vehicle classification is done on the computer, using decision trees. Prior to vehicle classification data detected by the laser scanner needs to be pre-treated. This pre-treatment consist of geometric corrections and detection and elimination of static objects on the road. The goal of data normalization is obtain a 3D matrix representing detected vehicle.

Data received from the laser scanner must be normalized in three steps:

- Geometric corrections, due to data measure by laser scanner.

- Elimination of static objects on the road.

- Lost reflections.

\subsection{Geometric corrections}

Data sent by laser scanner is the distance between the sensor and the vehicle or the detected object. This distance is not perpendicular to the road and is related with the angle, $\alpha$, at which the measurement is taken. Several authors refer to this consideration as geometric correction (Harlow, 2001). Graphically, in Fig. 3, value measured is A, whilst the information needed is the height $h$ and the position $x$. By simple mathematics the height of the object $h$ and the position $\mathrm{x}$ can be calculated.

\subsection{Static objects}

Detection and elimination of static objects on the road is easily implemented by storing an offset vector in absence of vehicles, Fig. 4. This offset vector is subtracted from the laser scanner data thus ensuring permanent fixtures and road anomalies are eliminated. 


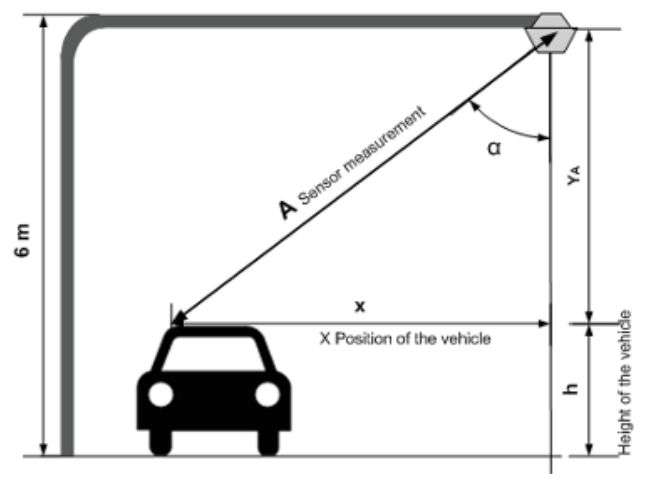

Fig. 3. Geometric Corrections

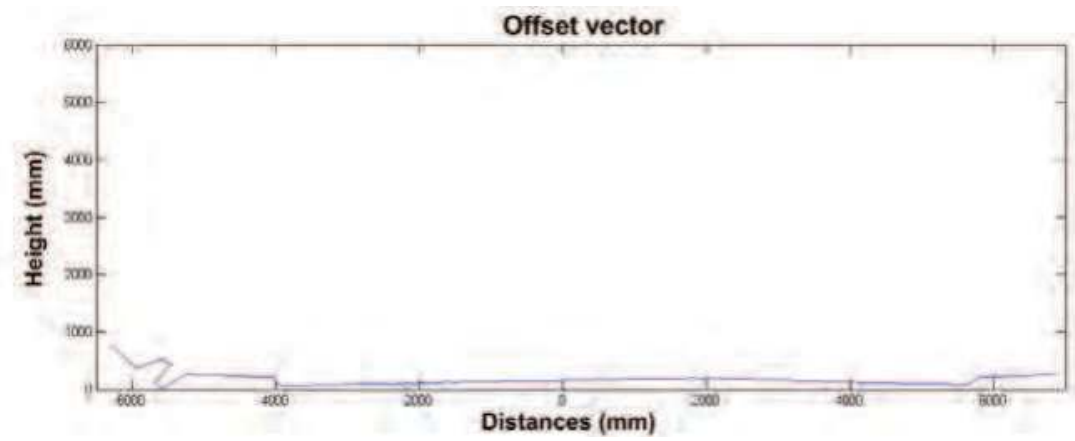

Fig. 4. Offset Vector

\subsection{Lost reflections}

The intensity of reflected sunlight from the road is a critical parameter that affects sensor measurements. Clouds blocking the sun are the most important cause for fast and/or large changes in intensity.

As explained previously, the laser scanner principle of operation is based on the measurement of the ToF. The changes in light intensity produce reflections too weak thus they are not received within the maximum detection time set by the sensor. These changes generate false measurements that can cause the sensor to report misleading data.

This effect is a characteristic of the vehicles most reflective parts i.e. the front and back windows. The light of the laser pulse emitted undergoes a reflection at an angle that does not agree with the detector position, thus the diffuse reflection does not take place in a direction that is advantageous to the detector. Fig. 5 shows the image of a vehicle with lost reflections that can be seen in the front and lateral windows because they show height values among 0 and $200 \mathrm{~mm}$, corresponding to the blue color. In the case of the front window, it is possible to verify the data as the expected received pulse values should be between 1.000 and $1.400 \mathrm{~mm}$, while what it is actually received are lower values due to the lost reflections.

In order to be able to correct these errors due to the intrinsic nature of the operation of the sensor it is necessary to do a depth revision of the detected values. After several studies, we 
conclude that in this situation all the erroneous values had a typical characteristic of height lower than one meter. Therefore, by filtering the information the erroneous data, so called lost reflections, are eliminated.

The following algorithm was used to eliminate the lost reflections:

- For each of the rows of the vehicle matrix:

- Step one: If there are unknown values between well known values, these known values are copied instead of each of the unknown values.

- Step two: If there are unknown values between two data sets with well known values, the greater values are copied instead of each of the unknown values.

- If there is a row in the matrix containing only unknown data, the row is left as it is.

- The matrix is transposed.

- For each row repeat steps one and two.

This algorithm is programmed in the DSP firmware. To reduce the processing time only the valid vehicle detection data is sent to the computer.

The corrections after executing the algorithm are shown in Fig. 5.

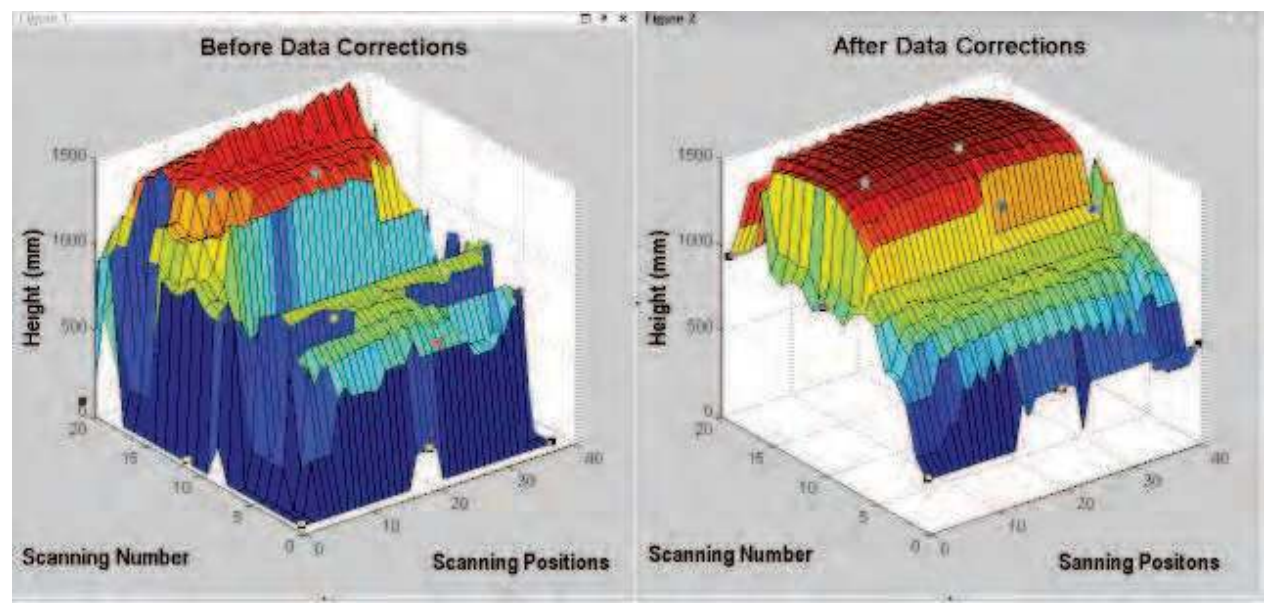

Fig. 5. Lost Reflections

\subsection{Data anomalies}

Laser scanner sensors that are mounted to the side or over a road may experience two types of data anomalies: lateral views and the effect of the speed (Klein et al., 2006).

Lateral view is an effect that can appear when a large vehicle hides another smaller one. The effect of tall cars blocking other lanes is shown graphically in Fig. 6. In this case a passenger car is almost hidden by a taller bus driving in an adjacent lane. The bus covers the passenger car from the sensor. The sensor detects the car, measurement A, but the next value detected, measurement $\mathrm{B}$, is for the bus, so both detections overlap and cause an undercounting. In order to determine overlaps and solve errors, it is necessary to analyze the matrix with the isolated vehicle information. 


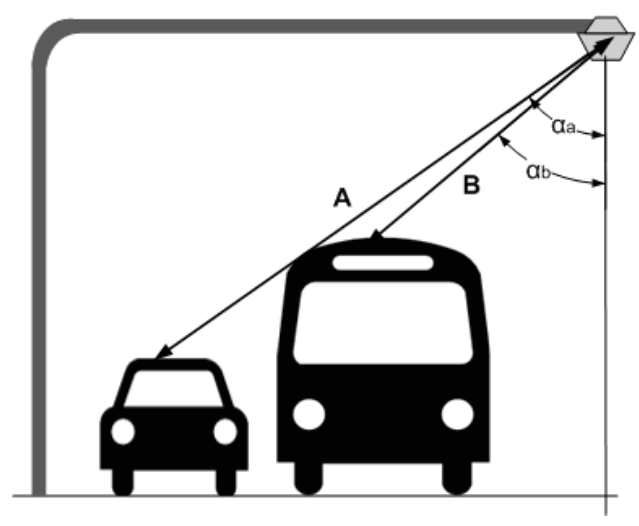

Fig. 6. Data Anomalies: Taller cars blocking angle's view

Also, the effect of speed has to be eliminated. Not all vehicles travel at the same speed on the road, so the information we have from each of them varies. This affects the silhouette obtained. By means of decimation and interpolation techniques, the matrices corresponding to all the vehicles which determinate their size are standardized. Thus, distortion effects due to the speed of travel are corrected.

\subsection{Detection techniques: string contour}

The detector covers not only one lane but four, so that more than one vehicle can be detected at a time, see Fig. 7(a). It is important to be able to isolate the different vehicles detected even though they have been detected at the same time. It is easy to reconstruct the vehicle when only one car crosses over the detection area. In this case all the information received belongs to the same car, and stream-by-stream the vehicle can be reconstructed. But in general, under normal circumstances this would not be the situation when several vehicles cross under the detector at the same time.

We used string contour techniques to isolate and reconstruct vehicles (Wilson, 1997), see Fig. 7(b) and Fig. 7(c). This technique makes it very simple and easy to implement the monitoring of contours in binary images. The basic function of the algorithm is to search, isolate and store the information of vehicles in independent files in a matrix format.

\subsection{Conclusions}

After a depth data normalization process information gathered by laser scanner can be isolated in a matrix. This matrix represents the vehicles detected once isolating one from others. Thus, the software requirements defined are shown in Table 2.

\section{Methods}

The classification algorithm aims to define a set of standards or group of patterns that allow the classification of the study group into two or more categories (Bishop, 2006; Jain et al., 2000). It was decided to use statistical recognition of standard systems because, from a set of numerical measures with well known probability distributions, recognition and classification is possible. 


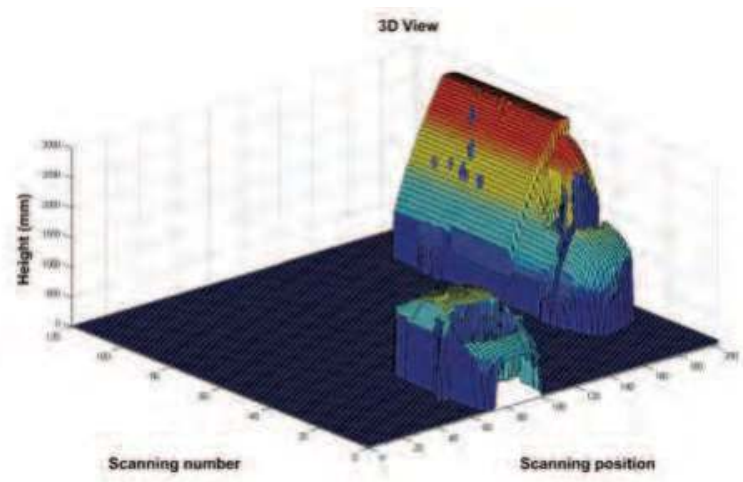

(a) $3 \mathrm{~d}$ view of the road

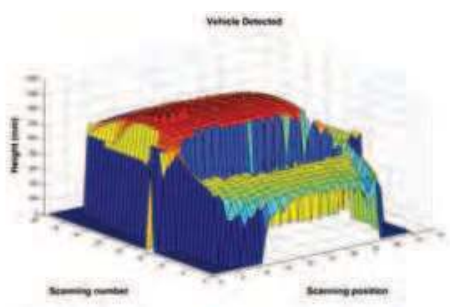

(b) Vehicle detected 1

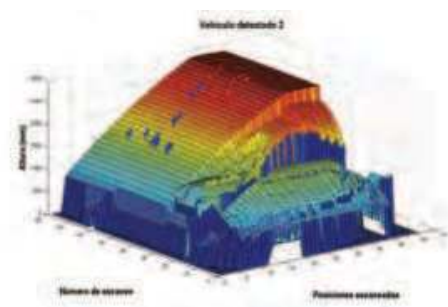

(c) Vehicle detected 2

Fig. 7. Detection techniques

\section{Software Requirements}

Data Acquisition

The system must be able to treat correctly the information sent by the laser scanner. DSP firmware must be able to carry data normalization by setting an offset line.

The system, after the correct data normalization, must sent these information to the computer for its processing via USB port at $1.5 \mathrm{MBd}$.

Data Treatment
Information received must be correctly treat in order to get valuable information
to be sent to the traffic control centers.
After this process the following information is due to obtain: vehicles detection and all
the statistical and values that can be inferred of them: intensity of the traffic (veh/h)
and density mainly.
The processing of the signal must follow the algorithms defined of data normalization
(geometric corrections, static objects and lost reflections) and data anomalies
(lateral views and the effect of the speed).
Information provided must be reliable and valuable.

Table 2. Software Requirements 
The classic techniques of patterns recognition and classification can be parametric (Bayesian, linear discriminant, support vector machine...) or nonparametric (histograms, K neighbours, decision trees ...). Since the 90's it is being use in addition techniques based on artificial neuronal networks. In the traffic area the neuronal networks have been used habitually in the case of artificial vision, with important congresses of IEEE dedicated to the subject. For example, the congresses on "Computer vision and Pattern Recognition" has already being celebrated since 1985. Multinomial pattern matching has been used in the detection of vehicles (Koch \& Malone, 2006) by means of IR or acoustic sensors (Cevher et al., 2007a;b).

After the data recollection, the first stage in the pattern recognition is the learning or training stage. In this phase it is used a group of data which, a priori, the class is known which they belong and this group serve to train to the system. This strategy denominates supervised learning, when knowing itself the patterns; if they were not known it would deal with learning non supervised (Friedman \& Kandel, 1999; Ripley, 1997).

In the cases of supervised learning two situations can be given: that the objective is to assign each value of entrance to one of a finite number of discreet categories, which is called classification problem; or that the objective is to assign one or more variable continuous, being then regression. The cases of supervised learning can have different objectives: to detect groups of similar examples within the data, clustering; to determine the distribution of the data in the entrance space, density estimation; or to project the data in spaces of smaller dimension to visualize them (Bishop, 2006).

The group of data known like training group, defines the patterns corresponding to each one of the categories that are desired to classify, and allows to determine the function that will be used for the classification (Bishop, 2006). The best the training group would be, the best the patterns will define and better discriminations will allow to carry out (Jain et al., 2000).

Once the model has been trained begins the second and last stage that is the classification or recognition of new data. This procedure is realized with the test group. In some practical applications where data are complex it is needed to pre-process itself to transform them into a new space of variables, so that the problem of recognition of pattern is easier to solve. The purpose of this stage of pre-processed (also called extraction of characteristics) is to find useful characteristics that are easy to calculate and that they maintain information for the discrimination in classes. That is to say, one is in charge to extract a set or vector of characteristics that will locate the data in points of the n-dimensional space of classification (Bishop, 2006; Sobreira \& Rodríguez, 2008). In this case the vector of characteristics is used like entrance of the algorithm of recognition of landlords. Also the pre-processed is realized if it is desired to increase the speed of calculation in applications of real time (Bishop, 2006).

\subsection{Classification: Decision trees \& boostraping}

Given a collection of records they are divided into two subgroups: a training group and a test group (Bishop, 2006). The classification algorithm is designed used decision trees. These trees are constructed beginning with the root of the tree and proceeding down to its leaves. Using decision trees we developed a model for each class attributes as a function of the values of other attributes (Matlab, 2009; Teknomo, 2009). Therefore future and unseen records could be assigned a category or class as accurately as possible. The test set is used to determine the accuracy of the model. 
The construction of decision trees is the method of supervised inductive learning more used. Its dominion of application is not restricted to a concrete scope but they can be used in diverse areas, from applications of medical diagnosis to games like the chess or systems of weather forecast (Díaz, 2007).

The decision trees adjust perfectly, as anticipated, to the data of the learning group, but they can realize predictions not very right in the case of new values. The branches inferiors, especially, can be seen strongly affected by atypical values. Simpler trees can offer often better results avoiding overfitting it (Matlab, 2009).

The technique of bootstrap is a resampling method that was proposed by Bradley Efron in 1979. It is used to approximate the distribution in the sampling of a statistical one. We used Boostraping techniques since boosting is a general method for improving the accuracy of any given learning algorithm (Michien et al., 1994). The concept of boosting, i.e. adaptive resampling, applies to many learning methods (Correa, 2004; Schapire \& Singer, 1999). However, resampling is particularly advantageous when used in conjunction with decision trees, because of two key reasons; decision tree algorithms are relatively efficient in high dimensions, and decision trees tend to have a bigger component of variance than other methods like nearest neighbors or neural nets (Apte et al., 1998).

\subsection{Classification standards}

Generally, the standards regarding vehicles classification do not have entity by themselves, but they are part and included in more extensive norms about ITS systems (Middlenton et al., 2002). The Department of American transport, by means of the family of standards NTCIP (acronym of National Transportation for Communication ITS Protocol) has defined the communication protocols and the vocabulary necessary to allow the interoperability between commercial equipment of traffic control of different manufacturers (NTCIP, 2009). Standard NTCIP 1209 deals with traffic sensors. It is in this standard where it will be included all regarding detection, count and classification of vehicles. In Europe, different standards for equipment have been developed from capture of data, but at national level, in countries like: France, Holland and Germany (Middlenton et al., 2002).

The number of axes of the heavy vehicles is also the base of the American standard defined by the FHWA (acronym of Federal Highway Administration) member of the American Department of Transport. The FHWA in its report "Traffic Monitoring Guide' ' it states: "for many of the carried out analyses by the traffic agencies the simple schemes of three groups (vehicles of passengers, articulated trucks of a unit and trucks) are valid, but other times a more sophisticated classification is required" (FHWA, 2001). At the moment, from the North American states are few that completely use the classification in 13 groups defined by the FHWA, although they use variations of the same following the vehicle park that circulates around each one of the states. In addition, the majority of the American manufacturers of commercial equipment already provides the classification of vehicles in the categories defined by the FHWA (Metrocount, 2009). The American standard is focused towards a classification centered in trucks, since of the 15 classes that define 6 of them they base on trucks with and without trailer and with diverse axes.

Finally, on Europe a great part of the counting equipment and commercial systems of classification are based on German standard TLS (defined by the federal institute of investigations of highways, Bundesanstalt fur Strassenwesen) (Bundesanstalt, 2008). 
Like in the American case, the most important companies of the sector also have adopted this standard. An example of it is: Weiss Electronics with models like the MC2024 (Electronic, 2009); Efkon with models like the AE TITAN 3000 (Efkon, 2009); and Xtralis with models like the DT 350 or TT290 (Xtralis, 2009). Also, European projects of VII Program Frame like project TRACKSS, use the same standard for applications of vehicle classification.

Although these classifications of vehicles are used by the administration, exist other that are not based on ITS applications. It is the case of the automobile companies, that use different classifications from their models in classes like: microcar, sedan, station wagon, sport cars, grand tourers ... They exist, also, the famous classifications of security Euro NCAP (acronym of European New Car Assessment Programme) that group among others to the vehicles in: supermini, family car, executive, MPV, $4 \times 4 \ldots$ (EuroNCAP, 2009).

The classification we used is done based on the German standard TLS. The classification is done in eight groups $(8+1)$ : powered two-wheels, passenger car, car with trailer, van, truck, truck with trailer, artic and bus, + unclassified. See Fig. 8.

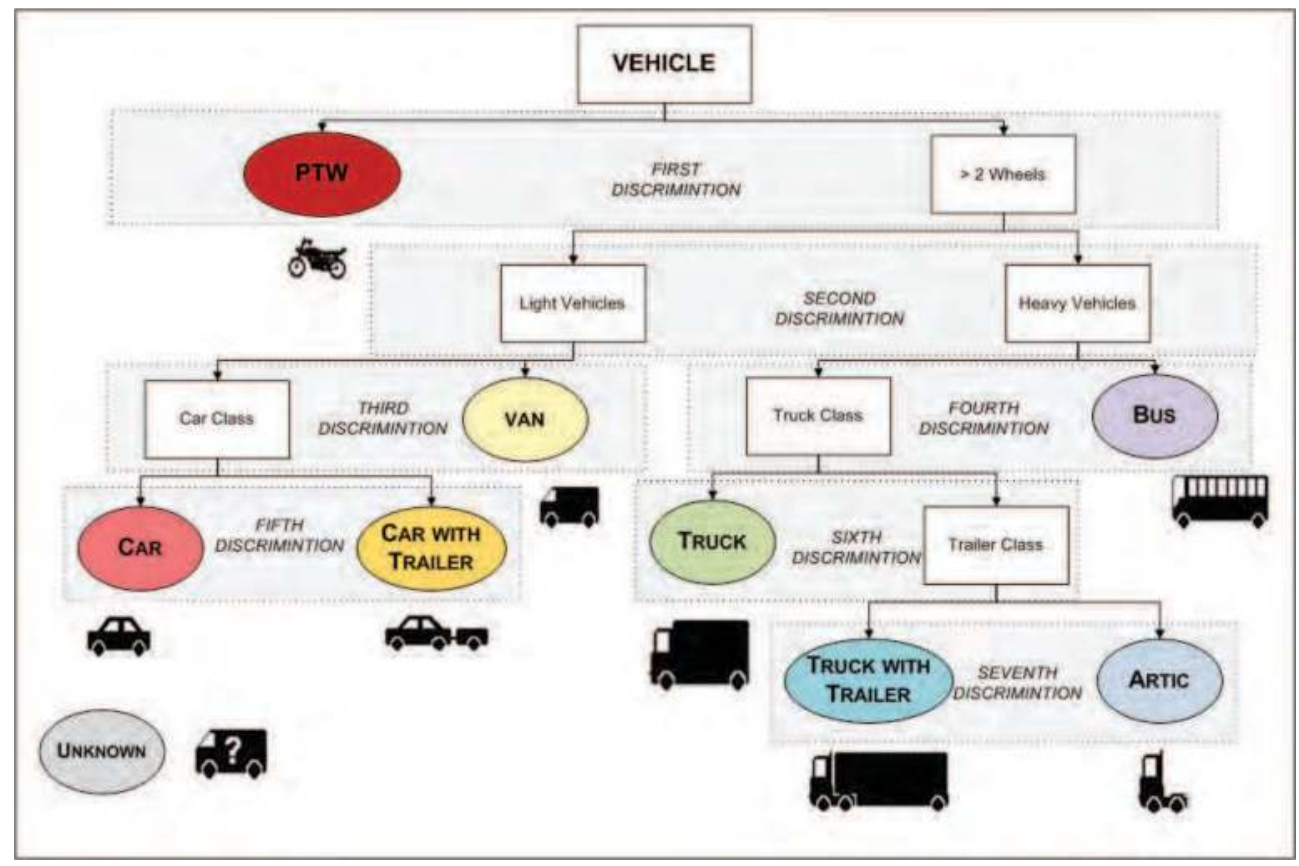

Fig. 8. Classification Tree

\section{Experimental results}

\subsection{Laboratory tests}

The detection and classification abilities of the prototype system were tested using a laboratory model in an environment as similar as possible to the road environment. The detector was placed 1.5 meters above the road instead of the 6 meters used in real conditions. Different vehicle prototypes were made on a 1:60 scale to the real ones. 
Initial detection tests were carried out with prototypes crossing the detector area every 2 seconds. In this situation, the vehicle detection worked successfully. The confusion matrix shows the counts of the actual versus predicted class values. It shows not only how well the model predicts, but also presents the details needed to see exactly where things may have gone wrong, and it is represented by:

- TP (True Positive) - number of vehicles detected $=495$.

- $F N($ False Negative) - number of vehicles not detected $=4$.

- FP (False Positive) - number of objects different from vehicles that have been detected $=1$.

- TN (True Negative) - number of objects different from vehicles that have not been detected $=0$.

\begin{tabular}{l} 
Evaluation from laboratory tests \\
\begin{tabular}{llll}
\hline Confusion Matrix & & \\
\hline Detection System & D & $\bar{D}$ \\
& D 495 & 1 \\
& $\bar{D}$ & 4 & 0 \\
\hline Detection Rate: $99.2 \%$ & & & \\
Precision: $99.8 \%$ \\
Accuracy: $99 \%$
\end{tabular} \\
\hline
\end{tabular}

Table 3. Laboratory Tests - Vehicle Detection

The system achieved high detection accuracy and precision in the case of vehicle detection with rates of $99.2 \%$ and $99.8 \%$, respectively. When the prototypes speed was increased the detection was also successfully.

The classification tests were done using the following protocol:

- The prototype A crosses the detection area every 10 seconds at a constant speed, for two minutes.

- Idem for prototype B.

- Prototypes A and B cross the detection area at the same time with similar speed every 10 seconds for two minutes.

The protocol was followed with different prototypes at different positions to verify the correct classification of vehicles. Five detections were not successful due to the fact that two cars were detected as one. Although this case was solved with modifications in the signal processing software, in terms of detection, it has to be considered as an error in the preliminary tests.

When prototypes are used, besides the problems caused by them all having the same scale, there is also the problem of the discrimination equations having to be modified proportionally to the scale used in them. Height and weight should be reduced for the correct use of the data extraction method therefore the classification was tested using a suitable database from previous projects. 


\subsection{Experimental tests}

The system was installed in the city of Valencia, Spain, on an urban road with two lanes of traffic in both directions. Thus, the system was tested with real urban traffic conditions. Also installed was a fixed camera next to the sensor to verify the information received and also to provide video images. The sensor is located on a post exclusively for this purpose. After the installation, minor adjustments were made to adapt the system to the characteristics of the road and the detection zone was delimited. The sensor was calibrated to correct possible misalignments during the installation. The system was installed outdoors for a period of six months. This period allowed testing the system in sunny conditions and at different times of the day.

First experiments were conducted at two different times of the day, early morning time between 8am and 11:30am, for "normal light value", and early afternoon period between 12noon and 2pm, for "high light intensity". The early morning is used for a reference when the sensor is known to be accurate. The early afternoon is used for testing the signal pre-treatment algorithm.

Several tests were carried out both in the "in the early morning" and "early afternoon" . All tests were successful at the communication level together with the signal acquisition and treatment software. Therefore, we can conclude that the detector work as expected in both light conditions.

The results of the experimental detection tests are shown in Table 4 . The indicators employed to evaluate each classification group are the standard evaluation metrics in case of detection given by: detection rate $97.9 \%$ (probability of vehicles from a particular group classified as such), precision $99.7 \%$ (probability of vehicles classified from a particular group that belong indeed to this group) and accuracy $97.6 \%$ (probability of vehicles classified correctly). The indicator precision penalizes false positive, it is to say 3 detections of irrelevant items in this case. Meanwhile detection rate penalizes the false negative of the system; that is, 21 vehicles not detected.

\begin{tabular}{llll}
\hline Confusion Matrix & & & \\
\hline Detection System & D & $\bar{D}$ \\
& D 976 & 3 \\
& $\bar{D}$ & 21 & 0 \\
\hline Detection Rate: $97.9 \%$ & & \\
Precision: $99.7 \%$ & & \\
Accuracy: $97.6 \%$ \\
\hline
\end{tabular}

Table 4. Experimental Tests - Vehicle Detection

Even though several experiments were carried out for classification, the results present are based on 972 vehicles detected: 51 powered two-wheels (2W), 675 passenger cars (PC), 178 vans $(\mathrm{V}), 37$ trucks $(\mathrm{T}), 7$ trucks with trailer (TT) and 24buses (B). The results in Table 5 show high accuracy in the case of vehicle classification with a rate of $94.24 \%$. Analysing the results one can conclude:

- The powered two-wheels are always classify correctly: 100\% recall and precision.

- The best detection ratio, after powered two-wheels, is the passenger car vehicles. Thus, $97.6 \%$ of the vehicles from this group have been detected correctly. 
- Errors in classification in the case of passenger car and van categories are due to the similarity between minivans and some passenger cars, mainly crossovers or category equivalent to the EuroNCAP class "Small Off-Roaders".

- The precision rate in the case of bus category is low, $81.5 \%$ due to the lack of sufficient samples.

- Because of the misclassifications of buses and trucks the recall rate in truck category is relatively low, $73.0 \%$.

- There were no car and trailer combinations in the tests.

\begin{tabular}{|c|c|c|c|c|c|c|}
\hline \multicolumn{7}{|c|}{ Evaluation from experimental tests } \\
\hline \multicolumn{7}{|c|}{ Confusion Matrix } \\
\hline & $2 \mathrm{~W}$ & PC & $\mathrm{V}$ & $\mathrm{T}$ & $\mathrm{TT}$ & $\mathrm{B}$ \\
\hline $2 \mathrm{~W}$ & 51 & 0 & 0 & 0 & 0 & 0 \\
\hline PC & 0 & 659 & 15 & 0 & 0 & 0 \\
\hline $\mathrm{V}$ & 0 & 15 & 151 & 4 & 0 & 1 \\
\hline $\mathrm{T}$ & 0 & 1 & 12 & 27 & 0 & 1 \\
\hline TT & 0 & 0 & 0 & 2 & 6 & 0 \\
\hline B & 0 & 0 & 0 & 4 & 1 & 22 \\
\hline \multicolumn{7}{|c|}{ Accuracy: $94.2 \%$ based on 972 vehicles detected } \\
\hline Classes & $2 \mathrm{~W}$ & PC & $\mathrm{V}$ & $\mathrm{T}$ & $\mathrm{TT}$ & B \\
\hline Recall $(\%)$ & 100 & 97.6 & 84.8 & 73.0 & 85.7 & 91.7 \\
\hline Precision $(\%)$ & 100 & 97.8 & 88.3 & 65.8 & 75.0 & 81.5 \\
\hline
\end{tabular}

Table 5. Experimental Tests - Vehicle Classification

\section{Conclusion}

Current Traffic Monitoring relies on information provided by the sensors installed in the road. This information is used both in real-time, to know what is happening on the roads, and in logs stored over long periods to have a global vision of the traffic over periods: of months or even years. The important aspects of the sensors is not only the parameters measured but also their reliability.

A laser scanner system for vehicle detection and classification, with high reliability, has been developed with a field of view able to cover up to 4 lanes. An original methodology for the treatment of the problem has been developed, with statistical rigor, from the normalization of the signals to silhouette generation. Preliminary tests presented good results with precision rate in detection of $97.9 \%$ and accuracy rate in classification of $94.2 \%$.

The system and method developed presents a valid option which is both simple and with a reduced computational cost. The system offers the possibility of using sensors that use the vehicles area detection principle with high accuracy in both detection and classification. Although the laboratory and field results validate the design, further tests will be performed to assess the impact of different weather conditions i.e. snow or rain. Meanwhile, a prototype is working at the Traffic Control Center of Valencia (Spain) to use in different traffic control applications such as cooperation between Laser Scanner and traffic lights for a better traffic distribution or restriction of vehicles to the city center. 


\section{References}

Abdelbaki, H., Hussain, K. \& Gelenbe, E. (2001). A laser intensity image based automatic vehicle classification system, Intelligent Transportation Systems, 2001. Proceedings. 2001 IEEE pp. 460-465.

Apte, C., Damerau, F. \& Weiss, S. (1998). Text mining with decision trees and decision rules, in C.-M. University (ed.), Conference on Automated Learning and Discovery.

Bishop, C. M. (2006). Pattern Recognition and Machine Learning, Information Science and Statics, Springer, New York.

Bundesanstalt für StraSSenwesen (2008). Technical delivery terms for route stations, TLS, Bundesanstalt für StraSSenwesen. [Online; Last Access October 2008].

URL: http://www.bast.de/nn43710/EN/eHome/ehomepagenode.html?nnn=true

Cevher, V., Chellappa, R. \& McClellan, J. (2007). Joint acoustic-video fingerprinting of vehicles, part i, Acoustics, Speech and Signal Processing, 2007. ICASSP 2007. IEEE International Conference on 2: 745-748.

Cevher, V., Guo, F., Sankaranarayanan, A. \& Chellappa, R. (2007). Joint acoustic-video fingerprinting of vehicles, part ii, Acoustics, Speech and Signal Processing, 2007. ICASSP 2007. IEEE International Conference on 2: 749-752.

Cheng, H. H., Shaw, B. D., Palen, J., Lin, B., Chen, B. \& Wang, Z. (2005). Development and field test of a laser-based nonintrusive detection system for identification of vehicles on the highway, Intelligent transportation systems, IEEE transactions on 6(2): 147-155.

Cheng, H., Shaw, B., Palen, J., Larson, J., Hu, X. \& Van katwyk, K. (2001). A real-time laser-based detection system for measurement of delineations of moving vehicles, Mechatronics, IEEE/ASME Transactions on 6(2): 170-187.

Correa, J. C. (2004). Método bayesiano bootstrap y una aplicación en la estimación del percentil 85 en ingeniería de tránsito, Revista Colombiana de Estadística 27(2): 99-107.

Díaz, Z. (2007). Predicción de crisis empresariales en seguros no vida, mediante árboles de decisión y reglas de clasificación, Colección: Línea 3000, Editorial Complutense.

Efkon (2009). Ae titan 3000, EFKON AG. [Online; Last Access August 2009].

URL: $h t t p: / / w w w . e f k o n . c o m / d o c s / T D s i A E T I T A N 01 E 03 . p d f$

Electronic, W. (2009). Classification detector mc2024, Weiss Electronic GmBH. [Online; Last Access August 2009].

URL: http://www.weiss-electronic.de/englisch/03-komponenten/03-03-03-

Ertico (2008). ITS Europe. [Online; Last Access November 2008].

URL: http://www.ertico.com/en/welcome_to_ertico_its_europe.htm

EuroNCAP (2009). Euro ncap vehicle types, European New Car Assessment Programme. [Online; Last Access January 2009].

URL: http://www.euroncap.com/

Fang, J., Meng, H., Zhang, H. \& Wang, X. (2007). A low-cost vehicle detection and classification system based on unmodulated continuous-wave radar, Intelligent Transportation Systems Conference, 2007. ITSC 2007. IEEE pp. 715-720.

FHWA (2001). Traffic monitoring guide. executive summary, Technical report, US Department of Transportation. Federal Highway Transportation. Office of Highway Policy Information. Report Number: FHWA-PL-01-021.

URL: http://www.fhwa.dot.gov/ohim/tmguide/

Friedman, M. \& Kandel, A. (1999). Introduction to Pattern Recognition: statistical, structural, neural and fuzzy logic approaches, Vol. 32 of Machine Perception and Artificial intelligence, World Scientific, Singapore. 
Fuerstenberg, K. \& Dietmayer, K. (2004). Object tracking and classification for multiple active safety and comfort applications using a multilayer laser scanner, Intelligent Vehicles Symposium, 2004 IEEE pp. 802-807.

Gallego, N., Mocholí, A., Menéndez, M. \& Barrales, R. (2007). Explotación de las infraestructuras actuales en entornos urbanos para aplicaciones its, VII congreso español en Sistemas de Inteligentes de Transporte, Valencia. .

Gallego, N., Mocholí, A. \& Menéndez, M. (2009). A real-time laser scanner intelligent sensor for its applications, Intelligent Transportation, 6th International Workshop on pp. 21-26.

García, L. A. (2000). Diseño e implementación de una arquitectura multiagente para la ayuda a la toma de decisiones en un sistema de control de tráfico urbano, $\mathrm{PhD}$ thesis, Departamento de Informática. Universidad Jaime I, Castellón de la Plana. España. Director: Dr. Francisco Toledo Lobo.

Harlow, C. (2001). Automatic vehicle classification system with range sensors, Transportation research. Part C: Emerging Technologies 9(4): 231-247.

Hussain, K. \& Moussa, G. (2005). Automatic vehicle classification system using range sensor, Information Technology: Coding and Computing, 2005. ITCC 2005. International Conference on 2: 107-112.

ITS (2008). ITS Spain. Ministry of Science and Innovation. [Online; Last Access November 2008].

URL: http://www.itsspain.com/itsspain/

Jain, A. K., Duin, R. P. W. \& Jianchang, M. (2000). Statistical pattern recognition: A review, Pattern Analysis and Machine Intelligence, IEEE Transactions on 22(1): 4-37.

Klein, L. A., Mills, M. K. \& Gibson, D. R. (2006). Traffic detector handbook, Technical report, US Department of Transportation. Federal Highway Transportation. Publication No. FHWA-HRT-06-108.

Koch, M. W. \& Malone, K. T. (2006). A sequential vehicle classifier for infrared video using multinomial pattern matching, Computer Vision and Pattern Recognition Workshop, 2006. CVPRW'06. Conference on pp. 127-127.

Martin, P. T., Feng, Y. \& Wang, X. (2003). Detector technology evaluation, Technical report, Department of Civil and Environmental Engineering University of Utah Traffic Lab. MPC Report number 03-154. [Online; Last Access November 2008].

URL: http://www.mountain-plains.org/pubs/html/mpc-03-154/

Matlab (2009). Statistics Toolbox 7. User's Guide, version 7.1 (released 2009b) edn.

Metrocount (2009). Traffic executive software, MetroCount. [Online; Last Access August 2009].

URL: http://www.metrocount.com/products/mte/index.html

Michien, D., Spiegelhalter, D. \& Taylor, C. (1994). Machine learning, neural and statistical classification.

Middlenton, D., Gopalakrishna, D. \& Raman, M. (December 2002). Advances in traffic data collection and management, white paper, Technical report, Office of Policy. Federal Highway Administration, Washington, DC. Work Order Number BAT-02-006.

Mimbela, L. E. \& Klein, L. A. (2007). A summary of vehicle detection and surveillance technologies used in intelligent transportation systems, Technical report, The Vehicle Detector Clearinghouse and US Department of Transportation. Federal Highway Transportation.

NTCIP (2009). National transportation communications for its protocol. [Online; Last Access August 2009].

URL: http://www.ntcip.org/info/ 
Palojarvi, P., Ruotsalainen, T. \& Kostamovaara, J. (2005). A 250-mhz bicmos receiver channel with leading edge timing discriminator for a pulsed time-of-flight laser rangefinder, Solid-State Circuits, IEEE Journal of 40(6): 1341-1349.

Peden, M., Scurfield, R., Sleet, D., Mohan, D., Hyder, A. A., Jarawan, E. \& Mathers, C. (2004). World Report on Road Traffic Injury Prevention, 1st edn, World Health Organization, Geneva, Switzerland.

Pehkonen, J., Palojarvi, P. \& Kostamovaara, J. (2006). Receiver channel with resonance-based timing detection for a laser range finder, Circuits and Systems I: Regular Papers, IEEE Transactions on 53(3): 569-577.

Ripley, B. D. (1997). Pattern Recognition and Neural Networks, Cambridge University Press, Cambridge, UK.

Sobreira, M. \& Rodríguez, A. (2008). Clasificación automática de fuentes de ruido de tráfico, Acústica 2008, V Congreso Ibérico de Acústica, 39ž Congreso Español de Acústica, Coimbra, Portugal .

Schapire, R. E. \& Singer, Y. (1999). Improving boosting algorithms using confidence-rated predictions, Machine Learning pp. 297-336.

Sepulcres, M. \& Gozálvez, J. (2006). Dimensionado de sistemas de comunicaciones móviles entre vehículos para aplicaciones de seguridad, Libro de Actas -URSI 2006. XI Simposium Nacional de la Unión Científica Internacional de Radio, 2006 pp. 1.158-1.161.

Skszek, S. L. (2001). State-of-the-art report on non-traditional traffic counting methods, Technical Report FHWAAZ-01-503, Arizona Department of Transportation.

Teknomo, K. (2009). Tutorial on decision tree. [Online; Last Access August 2009]. URL: http://people.revoledu.com/kardi/tutorial/decisiontree/

Turner, J. D. \& Austin, L. (2000). A review of current sensor technologies and applications within automotive and traffic control systems, Institute of the Mechanical Engineers, 2000. Proceedings IMechE 2000 214(D): 589-614.

UN (2003a). Resolution adopted by the general assembly: Global road safety crisis. $56^{t} h$ plenary meeting, United Nations. A/RES/58/9.

UN (2003b). Resolution adopted by the general assembly: Global road safety crisis. $86^{t} h$ plenary meeting, United Nations. A/RES/57/309.

UN (2004). Resolution adopted by the general assembly: Improving road safety. $84^{t} h$ plenary meeting, United Nations. A/RES/58/289.

UN (2005). Resolution adopted by the general assembly: Improving global road safety. $38^{t} h$ plenary meeting, World Health Assembly. A/RES/60/5.

UN (2008). Resolution adopted by the general assembly: Improving global road safety. $87^{t} h$ plenary meeting, World Health Assembly. A/RES/62/244.

UNFPA (2007). State of world population 2007.unleashing the potential of urban growth, United Nations Population Fund.

Van Arem, B., Van der Vlist, M., Blonk, J. \& Van den Berg, L. (1993). Demonstration of a general european road data information exchange network - gerdien, Vehicle Navigation and Information Systems Conference, 1993., Proceedings of the IEEE-IEE pp. 163-168.

WHA (2004). Resolution adopted by the $57^{t} h$ assembly: Road safety and health, World Health Assembly. WHA57.10.

Wilson, G. (1997). Properties of contour codes, Vision, Image and Signal Processing, IEE Proceedings - 144(3): 145-149.

Xtralis (2009). Traffic solutions, Xtralis. [Online; Last Access August 2009]. URL: $h$ ttp://xtralis.com $/ p . c f m ? s=22 p=381$ 


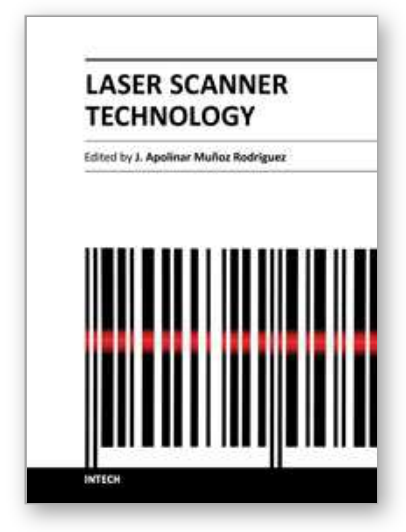

\author{
Laser Scanner Technology \\ Edited by Dr. J. Apolinar Munoz Rodriguez
}

ISBN 978-953-51-0280-9

Hard cover, 258 pages

Publisher InTech

Published online 28, March, 2012

Published in print edition March, 2012

Laser scanning technology plays an important role in the science and engineering arena. The aim of the scanning is usually to create a digital version of the object surface. Multiple scanning is sometimes performed via multiple cameras to obtain all slides of the scene under study. Usually, optical tests are used to elucidate the power of laser scanning technology in the modern industry and in the research laboratories. This book describes the recent contributions reported by laser scanning technology in different areas around the world. The main topics of laser scanning described in this volume include full body scanning, traffic management, 3D survey process, bridge monitoring, tracking of scanning, human sensing, three-dimensional modelling, glacier monitoring and digitizing heritage monuments.

\title{
How to reference
}

In order to correctly reference this scholarly work, feel free to copy and paste the following:

Nieves Gallego Ripoll (2012). Laser Scanner: eSafety \& ITS Applications, Laser Scanner Technology, Dr. J. Apolinar Munoz Rodriguez (Ed.), ISBN: 978-953-51-0280-9, InTech, Available from:

http://www.intechopen.com/books/laser-scanner-technology/laser-scanner-esafety-its-applications

\section{INTECH}

open science | open minds

\section{InTech Europe}

University Campus STeP Ri

Slavka Krautzeka 83/A

51000 Rijeka, Croatia

Phone: +385 (51) 770447

Fax: +385 (51) 686166

www.intechopen.com

\section{InTech China}

Unit 405, Office Block, Hotel Equatorial Shanghai

No.65, Yan An Road (West), Shanghai, 200040, China 中国上海市延安西路65号上海国际贵都大饭店办公楼 405 单元

Phone: +86-21-62489820

Fax: $+86-21-62489821$ 
(C) 2012 The Author(s). Licensee IntechOpen. This is an open access article distributed under the terms of the Creative Commons Attribution 3.0 License, which permits unrestricted use, distribution, and reproduction in any medium, provided the original work is properly cited. 